\title{
LEARNING FROM THE CHILDREN
}

M any times, I have heard pediatricians state that children are not small adults. It is therefore essential that children and those who care for them have their own set of guidelines for preventing and treating peritoneal dialysis (PD)-related infections.

The first set of guidelines for managing PD-related infections in children was published in 2000 (1). In the present supplement, updated and reorganized guidelines provide state-of-the-art information to the pediatric practitioner (2). Adult practitioners can learn much by reading these updated guidelines. An inspection of Table 1, which compares the guidelines of 2000 with those of 2012, shows how greatly expanded the new guidelines are.

Because of a paucity of research about the management of children with PD-related infections, authors of the earlier guidelines were forced to rely mostly on expert opinion. After publication of the last guidelines, leaders in caring for children on PD put together a consortium that established the International Pediatric Peritonitis Registry (IPPR). Data collected internationally under the auspices of that electronic registry form the basis for many of the new guidelines. However, many questions remain unanswered.

The format of the new guidelines shows many improvements. Suggestions appear as clear, succinct statements at the start of each guideline. The evidence is now graded as level 1 ("we recommend"), based on evidence of high (A) or moderate (B) quality, or as level 2 ("we suggest"), based on evidence of low (C) or very low (D) quality. "Not graded" is used for opinion-based suggestions. The work group continues to provide a rationale for each set of guidelines, followed by statements of limitations and research recommendations.

The guideline sections are well organized. Some of the new features of the 2012 guidelines are highlighted in the next few paragraphs.

In Sections 1 through 6, considerable emphasis is placed on the prevention of peritonitis in children. All of those sections, except for the one on prophylactic antibiotics (guideline 6 in the 2000 version), are new and mirror to a large extent sections in the International Society for Peritoneal Dialysis position statement on reducing the risks of PD-related infections in adults (3).
Table 2 of the guidelines outlines suggestions for PD training content. Also, an entire section is devoted to retraining because emerging data suggest that the material and procedures learned during the initial training are often modified or abandoned, which in some cases leads to peritonitis. This situation is highlighted as an area for future research.

The section on connectology is also new, and it succinctly summarizes the importance of connectology with respect to peritonitis risk. To prevent secondary fungal peritonitis, the prescription of oral nystatin or fluconazole in conjunction with antibiotic administration in PD patients is recommended with level $2 \mathrm{~B}$ evidence. Topical antibiotic prophylaxis for the catheter exit site is also recommended, with level $2 \mathrm{~B}$ evidence and with $\mathrm{a}$ suggestion for further studies comparing regimens of chronic exit-site care.

The subsequent sections (guidelines 8 through 22) outline the management of peritonitis and catheterrelated infections. In the 2000 guidelines, infection management appeared in guidelines 1-12, 14, 15.

The work group recommends that, ifintermittent doses of glycopeptide antibiotics (vancomycin or teicoplanin) are administered, blood levels of those antibiotics should be monitored in patients with residual kidney function (level 2A evidence). The work group does not recommend the intermittent administration of beta-lactam antibiotics. The reasons, which are based on data obtained from the IPPR and a European multicenter trial in children $(4,5)$, include delayed eradication compared with the eradication seen with continuous administration, and a higher rate of treatment failure in gram-negative peritonitis (level $1 \mathrm{~B}$ evidence). Automated PD, with its frequent rapid cycles, results in inadequate intraperitoneal antibiotic levels in many cases. The impact of those levels on repeat and relapsing peritonitis have not been well studied in either children or adults.

A new suggestion in the current guidelines, based on level 2 C evidence, is to use intraperitoneal cefepime as a single agent for empiric therapy. If cefepime is not available, then a first-generation cephalosporin combined with either ceftazidime or an aminoglycoside is recommended for initial therapy, reserving glycopeptide use (in addition to cefepime or other gram-negative coverage) 
TABLE 1

Comparison of 2000 and 2012 Pediatric Guidelines for the Prevention and Treatment of Peritoneal Dialysis (PD)-Related Infections

\begin{tabular}{ccc}
\hline Guideline & 2000 Version & 2012 Version \\
\hline 1 & Diagnosis of peritonitis & Training \\
2 & Empiric therapy of peritonitis & Automated PD modifications \\
3 & Gram-positive peritonitis & Catheter type and placement \\
4 & Gram-negative peritonitis & Early exit-site care \\
5 & Culture-negative peritonitis & Chronic exit-site care \\
6 & Fungal peritonitis & Connectology \\
7 & Evaluation of primary treatment response & Prophylactic antibiotic therapy \\
8 & Approach for those who fail to improve & Ostomy patients \\
9 & Relapsing peritonitis & Diagnosis of peritonitis \\
10 & Adjunctive therapy for peritonitis & Administration of antibiotics \\
11 & Catheter removal and replacement & Empiric antibiotic therapy \\
12 & Prophylactic antibiotic therapy & Gram-positive peritonitis \\
13 & Diagnosis of catheter exit-site infection & Gram-negative peritonitis \\
14 & Treatment of catheter exit-site infection & Culture-negative peritonitis \\
15 & & Fungal peritonitis \\
16 & & Relapsing peritonitis \\
17 & & Adjunctive therapy \\
18 & & Catheter removal and replacement \\
19 & & Diagnosis of catheter infections \\
20 & & Treatment of catheter infections \\
21 & & Modification of automated PD \\
22 & & Evaluation of primary response \\
Appendix A & & Failure to demonstrate improvement \\
Appendix B & & Terminology for peritonitis \\
Appendix C & Reporting of peritonitis rates \\
& & Drug delivery and stability \\
\hline
\end{tabular}

to centers in which more than $10 \%$ of Staphylococcus aureus isolates are methicillin- or oxacillin-resistant, or to patients with a history of methicillin-resistant S. aureus infection or colonization (level 2B evidence). For patients with a history of infection with ampicillinresistant vancomycin-resistant Enterococcus, linezolid should be used as part of initial empiric therapy. Notably, based on a randomized trial in patients in the intensive care unit (6), carbapenems are recommended if the effluent grows an organism with extended-spectrum beta-lactamase production.

Catheter removal plus antifungal antibiotic therapy is recommended for children with fungal peritonitis. The suggestion for catheter removal is stronger than it was in the 2000 guidelines, and it is buttressed by some newer literature on outcomes $(7,8)$. This approach has been the recommended practice in adults for some time, because it is considered to provide better protection to the peritoneal membrane and to preserve life. Antifungal antibiotic administration should continue for 2 weeks or longer after catheter removal and resolution of signs and symptoms of infection. Fluconazole is an appropriate antibiotic, with ease of administration and good peritoneal penetration. The work group suggests that a new PD catheter should not be placed for $2-3$ weeks after an existing catheter is removed for fungal, enteric, and refractory peritonitis (level $2 \mathrm{C}$ evidence). Few data are available in this area.

The definition of relapsing peritonitis is the same as that in the adult guidelines: a second peritonitis episode with the same organism, or a culture-negative episode that occurs within 4 weeks of completion of antibiotic treatment. The pediatric work group suggests giving an intraluminal fibrinolytic agent for the first relapse if there is no tunnel infection or intra-abdominal abscess to explain the relapse. That recommendation is based on studies using urokinase (intraluminal $1000 \mathrm{IU} / \mathrm{mL}$ or $5000 \mathrm{IU} / \mathrm{mL}$ ) or recombinant tissue plasminogen activator $(1 \mathrm{mg} / \mathrm{mL})$ to prevent relapsing or repeat peritonitis (level 2C evidence) (9-11). Notably, Williams et al. found that replacing the catheter instead of using urokinase therapy led to less repeat peritonitis (9). A specific 
recommendation is made to exchange the catheter once the effluent has cleared with antibiotic therapy if the relapsing episode is associated with tunnel infection and if the episode is a second occurrence of relapse.

Some other adjunctive therapies for the treatment of peritonitis are similar to those in the 2000 guidelines, with little supporting evidence. These adjunctive therapies include lowering the fill volume for $1-2$ days to ease pain (not graded), adding intraperitoneal heparin (500 - $1000 \mathrm{IU} / \mathrm{L})$ until the effluent clears (level 2B evidence), and administering immunoglobulin $\mathrm{G}$, either intraperitoneally or intravenously, to children with frequent refractory peritonitis or to infants who are known to be hypogammaglobulinemic and have peritonitis and sepsis. Some support for this latter guideline statement comes from a randomized controlled trial of intraperitoneal immunoglobulin during peritonitis in adults, which led to a more rapid fall in the effluent leukocyte count (12). This area is a promising one for future study.

Many children and adults are on automated PD rather than continuous ambulatory PD. Both the 2000 and the 2012 guidelines have sections on modifying automated PD during treatment for peritonitis, with some small changes having been made to the 2012 guidelines. For patients having little pain or for those using neutral-pH dialysis fluid containing bicarbonate (more "biocompatible" solutions), modifications may not be necessary. However, for those being given intermittent doses of intraperitoneal antibiotics, the new guideline recommends prolonging the dwell time to $3-6$ hours on the cycler for the duration of antibiotic therapy to permit re-entry of the agent into the peritoneal space between doses (level 2C evidence).

A new section of the 2012 guideline on ostomy patients will be particularly helpful to all clinicians caring for PD patients. I am often asked whether adult patients with ostomies can be safely placed on PD. Almost all the data on this subject are derived from the pediatric literature. Not infrequently, children require gastrostomy placement for feedings. To diminish the peritonitis risk, the pediatric work group provides five suggestions on management of such patients. Those suggestions include placing the exit site as far as possible from the ostomy; performing the gastrostomy before or at the time of placement of the PD catheter (rather than afterward); and for those already on PD, using an open surgical procedure for the gastrostomy, administering prophylactic antibiotics (including an antifungal) at the time of gastrostomy placement, and holding PD for a minimum of 1 day after placement. Although few data are available in this area, the pediatric PD team has much more experience in this area than the adult team does.
The appendices are an important part of the 2012 guideline.

The first appendix provides all-important definitions of the various types of peritonitis, including recurrent (a second episode within 4 weeks of completion of therapy for a prior episode, with a different organism), relapsing (a second episode within 4 weeks of completion of therapy for a prior episode, with the same organism or sterile), repeat (an episode that occurs more than 4 weeks after completion of therapy of a prior episode, with the same organism), refractory (failure of the effluent to clear after 5 days of appropriate antibiotics), and catheterrelated peritonitis (peritonitis in conjunction with an exit-site or tunnel infection, with the same organism or one site culture-negative). Those definitions mirror the adult guidelines. They are important in interpreting the literature and in calculating a center's peritonitis rate, because relapsing episodes are traditionally not included in the rate, but recurrent and repeat episodes are. The second appendix contains clear instructions on reporting peritonitis rates for a center, and the third provides important information on drug stability.

The 2012 guidelines should be closely read by everyone who cares for patients on PD. Those caring for adults on PD can learn from the experience with children. There is much in this guideline that can be applied to the adult population. The pediatric work group is to be congratulated on producing a set of guidelines that will improve the care of all patients on PD. Hopefully, the research recommendations also included will focus future efforts to expand knowledge about PD-related infections, because much remains to be learned about preventing and managing such infections in children and adults alike.

\section{DISCLOSURES}

The author has no financial conflicts of interest to declare.

Beth Piraino*

University of Pittsburgh

Pittsburgh, Pennsylvania, USA

*email: Piraino@pitt.edu

\section{REFERENCES}

1. Warady BA, Schaefer F, Holloway M, Alexander S, Kandert $M$, Piraino $B$, et al. on behalf of the International Society for Peritoneal Dialysis (ISPD) Advisory Committee on Peritonitis Management in Pediatric Patients. Consensus guidelines for the treatment of peritonitis in pediatric

This single copy is for your personal, non-commercial use only.

For permission to reprint multiple copies or to order presentation-ready copies

for distribution, contact Multimed Inc. at marketing@multi-med.com 
patients receiving peritoneal dialysis. Perit Dial Int 2000; 20: 610-24.

2. Warady BA, Bakkaloglu S, Newland J, Cantwell M, Verrina E, Neu $A$, et al. Consensus guidelines for the prevention and treatment of catheter-related infections and peritonitis in pediatric patients receiving peritoneal dialysis: 2012 update. Perit Dial Int 2012; 32(Suppl 2):S29-86.

3. Piraino B, Bernardini J, Brown E, Figueiredo A, Johnson DW, Lye WC, et al. ISPD position statement on reducing the risks of peritoneal dialysis-related infections. Perit Dial Int 2011; 31:614-30.

4. Zurowska A, Feneberg R, Warady BA, Zimmering M, Monteverde $M$, Testa $S$, et al. Gram-negative peritonitis in children undergoing long-term peritoneal dialysis. Am J Kidney Dis 2008; 51:455-62.

5. Schaefer F, Klaus G, Müller-Wiefel DE, Mehls 0. Intermittent versus continuous intraperitoneal glycopeptide/ ceftazidime treatment in children with peritoneal dialysis-associated peritonitis. The Mid-European Pediatric Peritoneal Dialysis Study Group (MEPPS). J Am Soc Nephrol 1999; 10:136-45.

6. Zanetti G, Bally F, Greub G, Garbino J, Kinge T, Lew D, et al. on behalf of the Cefepime Study Group. Cefepime versus imipenem-cilastatin for treatment of nosocomial pneumonia in intensive care unit patients: a multicenter, evaluator-blind, prospective, randomized study.
Antimicrob Agents Chemother 2003; 47:3442-7.

7. Miles R, Hawley CM, McDonald SP, Brown FG, Rosman JB, Wiggins KJ, et al. Predictors and outcomes of fungal peritonitis in peritoneal dialysis patients. Kidney Int 2009; 76:622-8.

8. Chang TI, Kim HW, Park JT, Lee DH, Lee JH, Yoo TH, et al. Early catheter removal improves patient survival in peritoneal dialysis patients with fungal peritonitis: results of ninety-four episodes of fungal peritonitis at a single center. Perit Dial Int 2011; 31:60-6.

9. Williams AJ, Boletis I, Johnson BF, Raftery AT, Cohen GL, Moorhead PJ, et al. Tenckhoff catheter replacement or intraperitoneal urokinase: a randomised trial in the management of recurrent continuous ambulatory peritoneal dialysis (CAPD) peritonitis. Perit Dial Int 1989; 9:65-7.

10. Klaus G, Schäfer F, Querfeld U, Soergel M, Wolf S, Mehls 0 . Treatment of relapsing peritonitis in pediatric patients on peritoneal dialysis. Adv Perit Dial 1992; 8:302-5.

11. Zorzanello MM, Fleming WJ, Prowant BE. Use of tissue plasminogen activator in peritoneal dialysis catheters: a literature review and one center's experience. Nephrol Nurs J 2004; 31:534-7.

12. Coban E, Ozdogan M, Tuncer M, Bozcuk H, Ersoy F. The value of low-dose intraperitoneal immunoglobulin administration in the treatment of peritoneal dialysis-related peritonitis. J Nephrol 2004; 17:427-30. 